\title{
Effect of isoproterenol, phenylephrine, and sodium nitroprusside on fundus pulsations in healthy volunteers
}

Leopold Schmetterer, Michael Wolzt, Alex Salomon, Alexander Rheinberger, Christian Unfried, Gabriele Zanaschka, Adolf Friedrich Fercher

\begin{abstract}
Aims/Background-Recently a laser interferometric method for topical measurement of fundus pulsations has been developed. Fundus pulsations in the macular region are caused by the inflow and outflow of blood into the choroid. The purpose of this work was to study the influence of a peripheral vasoconstricting (the $\alpha_{1}$ adrenoceptor agonist phenylephrine), a predominantly positive inotropic (the non-specific $\beta$ adrenoceptor agonist isoproterenol), and a non-specific vasodilating (sodium nitroprusside) model drug on ocular fundus pulsations to determine reproducibility and sensitivity of the method.
\end{abstract}

Methods-In a double masked randomised crossover study the drugs were administered in stepwise increasing doses to 10 male and nine female healthy volunteers. Systemic haemodynamic variables and fundus pulsations were measured at all infusion steps.

Results-Fundus pulsation increased during infusion of isoproterenol with statistical significance versus baseline at the lowest dose of $0 \cdot 1 \mu \mathrm{g} / \mathrm{min}$. Neither peripheral vasoconstriction nor peripheral vasodilatation affected the ocular fundus pulsations.

Conclusions-Measurements of fundus pulsations is a highly reproducible method in healthy subjects with low ametropy. Changes of local pulsatile ocular blood flow were detectable with our method following the infusion of isoproterenol. As systemic pharmacological vasodilatation or vasoconstriction did not change fundus pulsations, further experimental work has to be done to evaluate the sensitivity of the laser interferometric fundus pulsation measurement in various eye diseases.

(Br f Ophthalmol 1996; 80: 217-223)

Although $85 \%$ of the blood volume in the eye circulates in the choroid, ${ }^{1}$ choroidal circulation is far less investigated than the retinal blood flow. The outer layers of the retina are nourished by the choroid, and animal experiments suggest that $90 \%$ of the oxygenation of the photoreceptors are supplied by choroidal circulation. ${ }^{2}$ The introduction of fluorescein angiography, ${ }^{3}$ particularly of video fluorescein angiography, ${ }^{4}$ has increased the understanding of choroidal circulation. However, systematic investigations of choroidal haemodynamics have not yet been carried out and quantitative pressure flow relations in human choroidal vessels are as yet unknown. Linear choroidal pressure flow relations have been obtained in animal experiments in different species. ${ }^{5-8}$ In the rabbit, the choroidal blood flow has been shown to be pressure independent when IOP was less than $20-25 \mathrm{~mm} \mathrm{Hg}$. 9

Blood vessels can be considered as cylinders filled with fluid at a pressure greater than that outside the cylinders. The pressure difference between the inside and the outside of a vessel is called the transmural pressure $P$. The corresponding tension $T$ in the vessel wall can be calculated by Laplace's law $P=T / R$, where $R$ is the radius of the cylinder. Any variation of the transmural pressure alters the tension of the vessel wall. Thus, pressure oscillations of cardiac pulse pressure output lead to a pulsation of the vessel wall. As blood is pumped into an artery, the systolic pressure increases and dilates the vessel wall. When cardiac ejection decreases, the pressure falls and the vessel wall returns to its equilibrium position, the diastolic pressure. ${ }^{10}$ The arterial pressure contour becomes progressively more distorted as the wave is transmitted down the arterial system. The elastic properties of an artery are described by the arterial compliance, the change in diameter over the change in pressure. The non-linear elastic response of arteries implies that their mechanical properties depend on the mean arterial pressure. ${ }^{11}$

In retinal and choroidal vessels the mean transmural pressure at the arteries entering the eye nearly equals the mean perfusion pressure, defined as the pressure in the arteries entering the eye $(\mathrm{Pa})$ minus the pressure in the veins (Pv) leaving the eye. $\mathrm{Pf}=\mathrm{Pa}-\mathrm{Pv}$. The pressure in the veins, $\mathrm{Pv}$, is significantly higher than the intraocular pressure (IOP) in the retinal vessels ${ }^{12}$ whereas the difference in the choroidal vessels is small. ${ }^{13}$ Therefore, the transmural pressure in the veins is rather small or even zero. Changes in transmural pressure and the consecutive change in vessel diameter lead to pulsations of the surrounding tissue.

Our work is concerned with local tissue pulsations at the foveola. This area of highest visual acuity is approximately $350 \mu \mathrm{m}$ in diameter. ${ }^{14}$ This is a little smaller than the retinal avascular zone measuring approximately $500-600 \mu \mathrm{m}$ in diameter. In our study, where the area involved in the measurement is $20-50$ $\mu \mathrm{m}$, the effect of tissue pulsations is only influenced by the blood flow in choroidal vessels. 
Hence the inflow and outflow of blood into the choroid leads to pulsatile movements of the eye fundus.

These fundus pulsations are measured with a recently described laser interferometer, ${ }^{15}$ which measures the distance changes between cornea and retina. The purpose of this study was to evaluate the short term variability, the day to day variability, the intersubject variability, and the sensitivity of the method to detect changes in ocular blood flow in healthy volunteers. We have therefore studied the effects of well characterised cardiovascular model drugs on fundus pulsations in a double masked randomised placebo controlled crossover study.

\section{Materials and methods}

In a double masked randomised crossover study design, subjects were assigned to receive infusions of stepwise increasing doses of a peripheral vasoconstricting, a peripheral vasodilating, a predominately positive inotropic drug, and of placebo (physiological saline solution) on different study days. From the 24 possible sequences of administration in the four way crossover design we randomly chose 19 .

\section{DRUGS ADMINISTERED}

These were phenylephrine (Neosynephrine, Winthrop Breon Laboratories, NY, USA; dose $0.5,1,2,4,8,16 \mu \mathrm{g} / \mathrm{kg} / \mathrm{min}$ ), sodium nitroprusside (Nipruss-Trockensubstanz zur Infusionsbereitung, Sanol-Schwarz, Monheim, Germany; dose $0.5,1,2,4,8,16 \mu \mathrm{g} / \mathrm{kg} / \mathrm{min})$, isoproterenol (Isuprel, Winthrop Breon Laboratories, NY, USA; dose $0 \cdot 1,0.2,0.4$, $0 \cdot 8,1 \cdot 6,3 \cdot 2 \mu \mathrm{g} / \mathrm{min}$ ), physiological saline solution. The drugs were diluted with saline or glucose (for sodium nitroprusside) to yield appropriate concentrations.

\section{PATIENTS}

Ten men (aged 20-33 years, mean 25.6 (SD 3.7) and nine women (aged 20-30 years, mean $25 \cdot 0(3.6)$ gave written informed consent

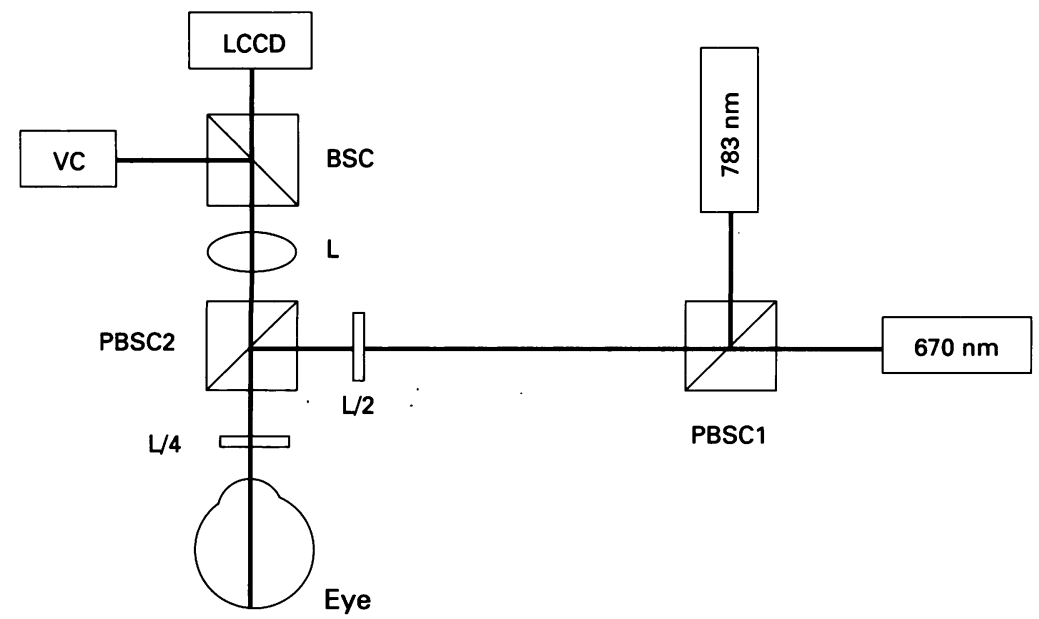

Figure 1 Optical scheme of the laser interferometer. (PBSC=polarising beam splitter cube $B S C=$ beam splitter cube, $L / 2=$ half wave plate, $L / 4=$ quarter wave plate, $L=$ lens, $V C=$ video camera, LCCD=linear charge coupled device array). to participate in this study according to procedures approved by the ethics committee of Vienna University School of Medicine. All subjects were drug-free, non-smokers and judged to have no evidence of any relevant physical disorder. All had normal laboratory evaluations, normal results from ultrasound examinations of carotid arterial blood flow, and normal findings from ophthalmic examinations with ametropy of less than $2 \cdot 5$ dioptres and intraocular pressure in the range of 11 to $16 \mathrm{~mm} \mathrm{Hg}$. The testing of the women was scheduled between the third and eighth day of menstrual cycles; pregnancy was excluded by urine pregnancy test (hCG Urine+Plus, Abbott, Wiesbaden, Germany).

All subjects were asked to refrain from alcohol and caffeine for at least $\mathbf{1 2}$ hours before study days. Initially, a 20 gauge plastic cannula (Venflon, Viggo-Spectramed, Helsingborg, Sweden) was inserted into a suitable antecubital vein, and a continuous infusion $(1 \mathrm{ml} / \mathrm{min})$ of physiological saline was started for baseline measurements to the comfortably sitting subjects. Each stepwise infusion period lasted 10 minutes unless there was an increase of $>40 \mathrm{~mm} \mathrm{Hg}$ or decrease of $>20 \mathrm{~mm} \mathrm{Hg}$ in mean arterial pressure, or until an increase of $>40$ per minute or decrease of $>20$ per minute in heart rate versus baseline infusion period, or until any systemic effects - for example, palpitation, headache, or dizziness occurred. Measurements of fundus pulsations were performed in the last 5 minutes of each infusion step.

In addition, we conducted measurements to assess the influence of isoproterenol and phenylephrine on IOP of eight male volunteers in our study group. Baseline measurements of IOP and fundus pulsations were performed during a 10 minute infusion of physiological saline solution. The drug effect was calculated from measurements of 10 minute infusions of $0.8 \mu \mathrm{g} / \mathrm{min}$ isoproterenol and of $2 \mu \mathrm{g} / \mathrm{kg} / \mathrm{min}$ phenylephrine, respectively. Two measurements of IOP and fundus pulsation measurements were performed during the last 5 minutes of each infusion step with a Goldmann applanation tonometer. No measurements of the effect of sodium nitroprusside on IOP were performed, since previous studies indicate that the influence of systemic administration of sodium nitroprusside $^{16}$ on IOP is small. In contrast, topical administration has been represented to lower IOP effectively. ${ }^{17}$

Systolic and diastolic blood pressures were measured by an automated oscillometric device (HP CMS patient monitor, Hewlett Packard, Palo Alto, CA, USA). Mean arterial pressure was calculated as two thirds diastolic pressure plus one third systolic pressure; pulse pressure amplitude was calculated as systolic blood pressure minus diastolic blood pressure. Pulse rate was registered automatically from a finger pulse oximetric device (HP CMS patient monitor).

Measurements of fundus pulsations were performed with a laser interferometric technique, which has been described in detail elsewhere. ${ }^{15}$ The interferometer, schematically 
Coefficients of variation (\%)

Short term variability

Day to day variability

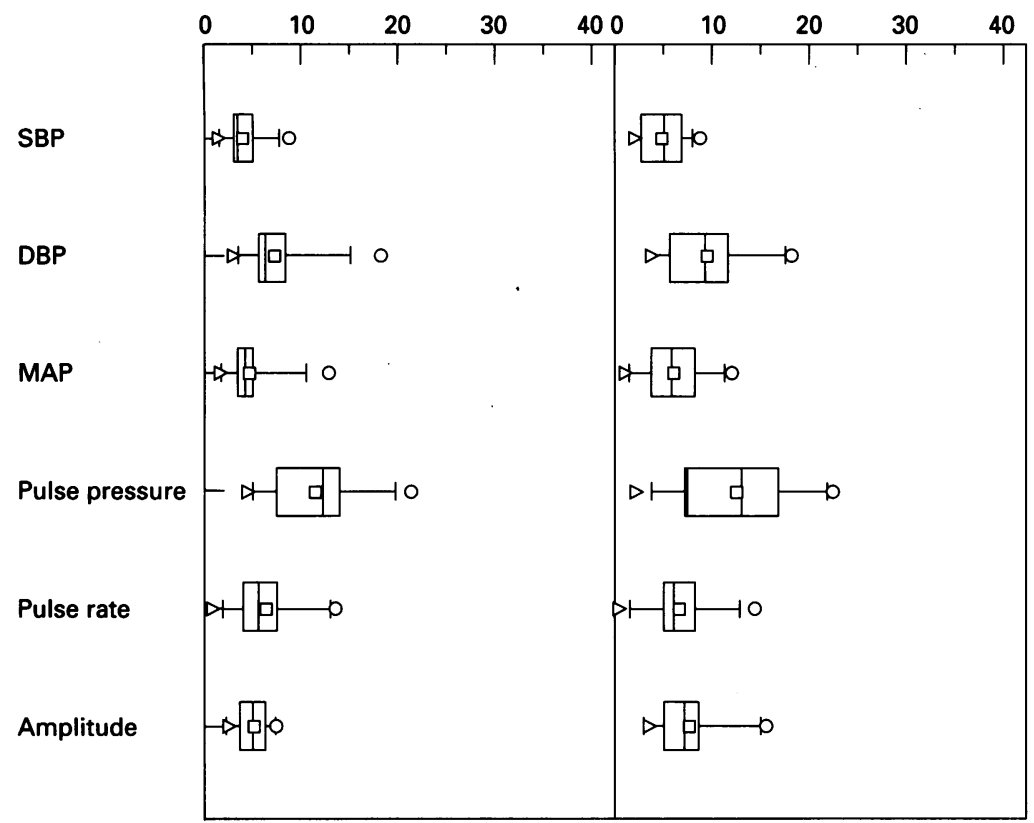

Figure 2 Short term (left panel) and day to day (right panel) variability of our measurements in 19 healthy subjects. In the right panel coefficients of variation were calculated for any subject from the four baseline recordings of the 4 study days. In the left panel they were calculated from the seven recordings of saline infusion study day. The bottom of the vertical box line marks $25 \%$, the median $50 \%$, and the top of the box $75 \%$. Whiskers represent $5 \%$ and $95 \%$. Mean (square symbols), minimum (triangles), and maximum values (circles) are presented. ( $S B P=$ systolic blood pressure, $D B P=$ diastolic blood pressure, $M A P=$ mean arterial pressure, pulse pressure $=$ pulse pressure amplitude calculated from $S B P-D B P$, amplitude $=$ fundus pulsation amplitude.

shown in Figure 1, is a simplified version of the instrument described by Schmetterer ${ }^{15}$ based on the same principle. A single mode laser diode (Sharp LT $023 \mathrm{MD0} ; \lambda=783 \mathrm{~nm}$ ) emits the beam used for the measurement. The beam of a second laser diode (Toshiba TOLD 9211; $\lambda=670 \mathrm{~nm}$ ) is used to adjust the beam into the patient's eye and is switched off during the measurement. The polarising beam splitter PBSC1 combines these two beams to a single beam of approximately $1 \mathrm{~mm}$ diameter illuminating the subject's eye. The beam passes a half wave plate which defines the polarising state. Then the polarising beam splitter PBSC2 deviates the beam towards the subject's eye. This beam splitter is used together with a quarter wave plate to reduce reflective losses in the light re-emitted from the eye when it passes towards the detecting unit. The beam is reflected both at the front of the cornea and at the retina.

These two reflected beams generate nonlocalised concentric circular interference fringes. As the path difference between the two interfering beams is twice the optical length $L$ of the eye, the interference order $\mathrm{N}=2 \times \mathrm{L} / \lambda$ is a very large number. Distance variations between cornea and retina lead to a corresponding variation $\Delta \mathrm{N}(\mathrm{t})$ of the interference order. $\Delta N(t)$ equals the number of fringes that have moved up to the moment $t$ through a fixed point in the interferogram. The time course of the optical distance variation $\Delta L(t)$ is:

$$
\Delta \mathrm{L}(\mathrm{t})=\Delta \mathrm{N}(\mathrm{t}) \times \lambda / 2
$$

The detecting unit comprises a linear charge coupled device (CCD) array and a video camera. Owing to the high spatial coherence of the illuminating laser beam, the interferences of the two re-emitted beams are not localised. Optimal visibility can only be achieved in a plane approximately $40 \mathrm{~mm}$ in front of the eye. This plane is imaged by the lens onto the CCD array and via a beam splitter cube (BSC) onto the photo cathode of the video camera (VC). The video camera is used to supervise the measurement procedure on a video monitor.

The readout of the CCD array is a spatiotemporal display of the fundus pulsations. Counting the number of fringes moving inwards and outwards the fundus pulsation amplitude (FPA), which is the maximum distance change between cornea and retina during the cardiac cycle, can be calculated. The FPA represents the difference of blood volume during the systole and diastole at one particular point of the fundus.

A headrest is used to fix the subject's head. The whole instrument is mounted on two translation stages and can be moved in $x$ and $y$ direction perpendicular to the optical axis of the subject's eye by computer controlled stepper motors. The observation of the visible laser beam reflected at the outer surfaces of the eye and the eyelid facilitates the gross adjustment of the instrument.

\section{STATISTICAL ANALYSIS}

Statistical analysis was done with Statistica software package (StatSoft Inc, Tulsa, OK, USA). Day to day reproducibility was calculated from the baseline registrations of the 4 study days. Using the seven measurements of the 10 minute saline infusion periods we calculated the short term variability. For each subject the

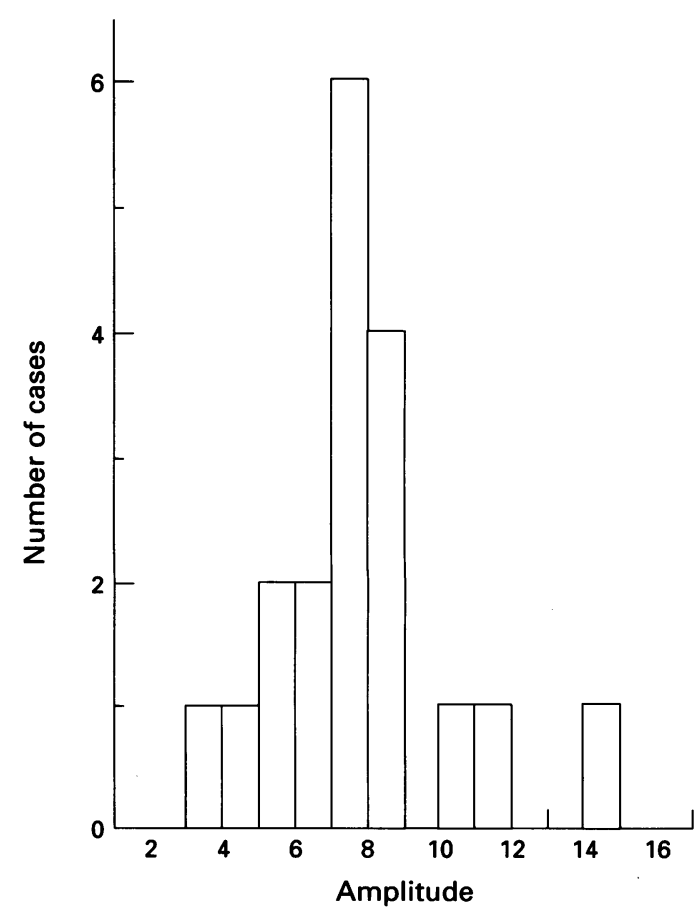

Figure 3 Results of fundus pulsation measurements in 19 healthy subjects under baseline conditions. The amplitude denotes the fundus pulsation amplitude in units of $\lambda / 2$. 


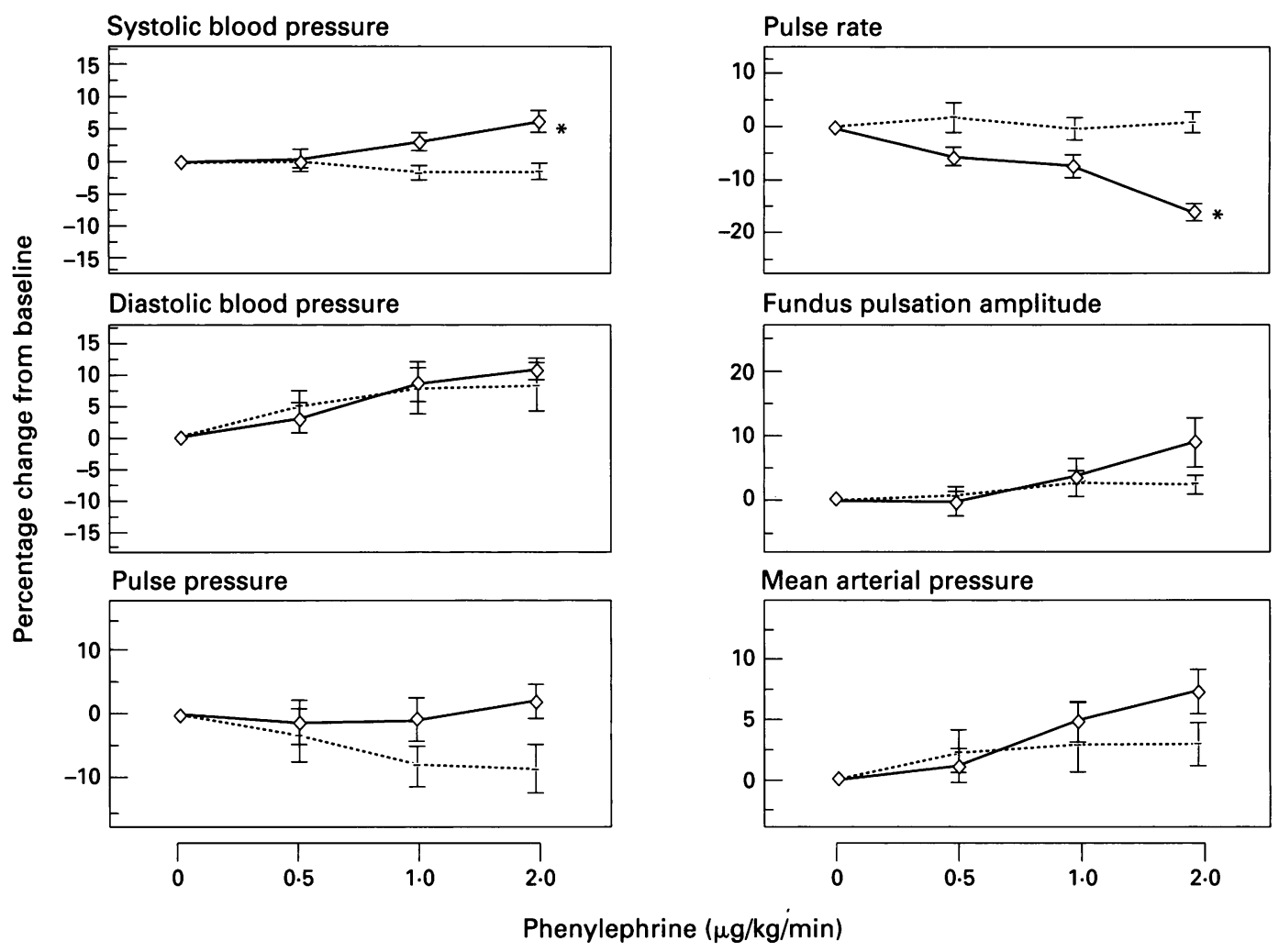

Figure 4 Dose-response relation (solid line) of changes (\%) from baseline measurements of systemic haemodynamics and of fundus pulsations during saline ( 0 ) and stepwise infusion of phenylephrine at doses of $0.5,1.0$, and $2.0 \mu \mathrm{g} / \mathrm{kg} / \mathrm{min}$. The broken line presents the results from the placebo study day. The asterisks indicate significant treatment effects versus placebo as calculated by repeated measure ANOVA $(p<0 \cdot 05)$. Results are presented as means (SEM).

coefficient of variation of measurements was calculated.

Results of fundus pulsation measurements were expressed as percentage change from the baseline. Significance was calculated versus baseline by repeated measure ANOVA, and versus placebo by two way repeated measure ANOVA. For these purposes only infusion steps with less than $20 \%$ drop outs were taken into consideration. The significance level was set to $p=0.05$. Significances are presented as $\mathrm{p}<0.05$ or $\mathrm{p}<0.005$.

\section{Results}

Results for day to day and short term variability are shown in Figure 2. Interindividual differences are shown in Figure 3.

PHYSIOLOGICAL SALINE SOLUTION

No time dependence of the seven short term readouts was detected.

\section{PHENYLEPHRINE}

In three subjects the infusion was stopped at 2 $\mu \mathrm{g} / \mathrm{kg} / \mathrm{min}$, in 10 at $4 \mu \mathrm{g} / \mathrm{kg} / \mathrm{min}$, in four at 8 $\mu \mathrm{g} / \mathrm{kg} / \mathrm{min}$, and in two at $16 \mu \mathrm{g} / \mathrm{kg} / \mathrm{min}$. There was a small increase in systolic $(p<0.05$ versus placebo and baseline), diastolic (NS versus placebo, $\mathrm{p}<0.05$ versus baseline), and mean arterial blood pressure (NS versus placebo, $\mathrm{p}<0.05$ versus baseline). The pulse pressure amplitude was nearly constant during the infusion steps. The pulse rate decreased dose dependently $(p<0.005$ versus placebo and baseline). The amplitude of fundus pulsations showed a small increase, which did not reach statistical significance (Fig 4).

ISOPROTERENOL

The infusion was stopped at $1.6 \mu \mathrm{g} / \mathrm{min}$ in eight subjects and at $3 \cdot 2 \mu \mathrm{g} / \mathrm{min}$ in seven subjects. In the remaining four subjects all seven infusion steps were administered. The systolic blood pressure $(p<0.005$ versus placebo and baseline) and the pulse pressure amplitude $(p<0.005$ versus placebo and baseline) increased significantly. The mean arterial pressure and the diastolic blood pressure did not change. The pulse rate increased significantly ( $p<0.005$ versus placebo and baseline). The fundus pulsation amplitude increased dose dependently $(p<0.005$ versus baseline and placebo, Fig 5).

SODIUM NITROPRUSSIDE

At $4 \mu \mathrm{g} / \mathrm{kg} / \mathrm{min}$ the infusion was stopped in six subjects, at $8 \mu \mathrm{g} / \mathrm{kg} / \mathrm{min}$ in eight subjects, and at $16 \mu \mathrm{g} / \mathrm{kg} / \mathrm{min}$ in five subjects. Blood pressure decreased during the infusion of sodium nitroprusside: systolic pressure $(p<0.05$ versus placebo and $p<0.005$ versus baseline), diastolic pressure $(p<0.005$ versus baseline and placebo), and mean arterial pressure $(\mathrm{p}<0.005$ versus baseline and placebo). Pulse rate increased significantly (NS versus placebo, $p<0.05$ versus baseline), whereas pulse pressure amplitude decreased only 


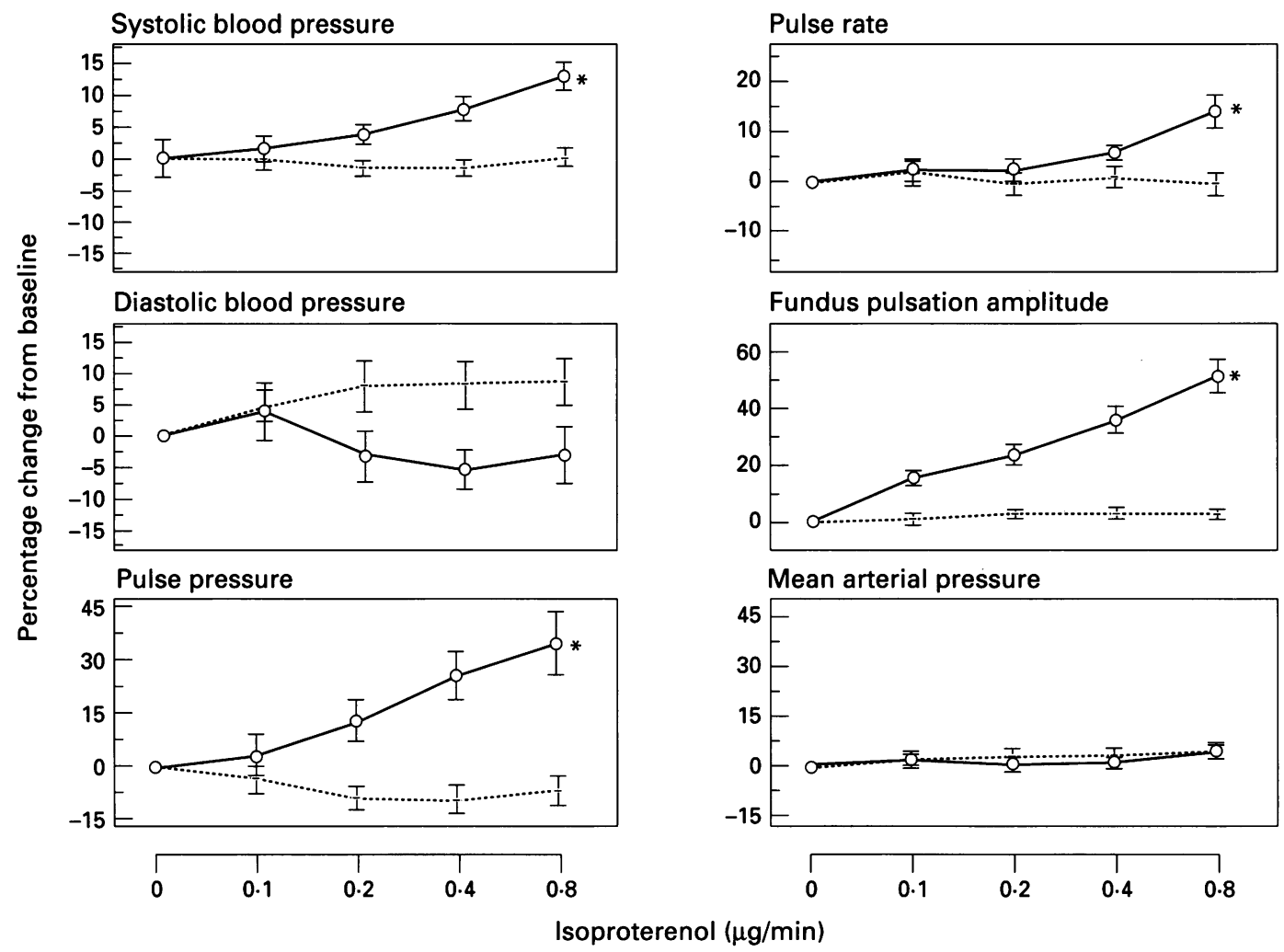

Figure 5 Dose-response relation (solid line) of changes (\%) from baseline measurements of systemic haemodynamics and of fundus pulsations during saline ( $(0)$ and stepwise infusion of isoproterenol at doses of $0 \cdot 1,0 \cdot 2,0.4$, and $0.8 \mu \mathrm{g} / \mathrm{min}$. The broken line presents the results from the placebo study day. The asterisks indicate significant treatment effects versus placebo as calculated by repeated measure ANOVA $(p<0 \cdot 05)$. Results are presented as means $(S E M)$.

slightly during the infusion steps. Fundus pulsations did not show significant changes during the infusion steps (Fig 6).

\section{IOP MEASUREMENTS}

The results of our IOP measurements during isoproterenol and phenylephrine infusion are summarised in Table 1 . No changes in IOP during administration of isoproterenol and phenylephrine were observed.

\section{Discussion}

Our results show that fundus pulsations in normal volunteers with ametropy of less than plus or minus $2 \cdot 5$ dioptres are dose dependently increased during infusion of isoproterenol but not during infusion of phenylephrine or sodium nitroprusside. Even at higher doses of the peripheral vasoconstrictor and the vasodilator the changes in the amplitude of fundus pulsations are small.

The intrasubject variability of this novel method is generally small. The within day variability, calculated from the saline infusion study day, was slightly smaller than the day to day variability, calculated from the baseline registrations of the 4 study days. This finding was also observed for the haemodynamic variables and argues for slightly different cardiovascular reactivity on different study days.

The intersubject variability of our measurements is much wider and may be caused by several ocular variables. James et $a l^{18}$ observed a significant change of the ocular pulse with axial eye length and refractive state of the eye.
In our measurements the influence of bulbus length is probably low owing to the small refractive errors of our study group. Moreover, the axial eye length mainly influences the propagation of the pulse wave through the eye. Intersubject differences in the angioarchitecture of the choroid, especially the organisation of the vessels in the submacular region, as reported by Fryczowski et al, ${ }^{19}$ may have an important effect on our measurements. A decrease of the ocular pulse with an increasing heart rate was reported for patients with pacemakers. ${ }^{20}$ These findings suggest that, at an increase of the heart rate, there may be a shift from pulsatile to non-pulsatile blood flow in the ocular circulation. ${ }^{20}$

During isoproterenol infusion the positive inotropic effect was responsible for both the dose dependent increase in pulse pressure amplitude and fundus pulsation amplitude. A direct comparison of the two variables is not possible as we do not know the relation between changes in systemic blood pressure and the perfusion pressure in the ocular vessels. Although the presence of $\beta$ adrenergic receptors in choroidal vessels was recently assumed by showing an increased choroidal vascular tone after systemic administration of timolol maleate, ${ }^{21}$ it is obvious that our observations mainly depend on the cardiovascular effects than on the confounding vasoconstricting or vasodilating vascular responses.

The mean arterial pressure was significantly changed following administration of phenylephrine and sodium nitroprusside, whereas both drugs did not affect pulse pressure amplitude. When considering the results after the 


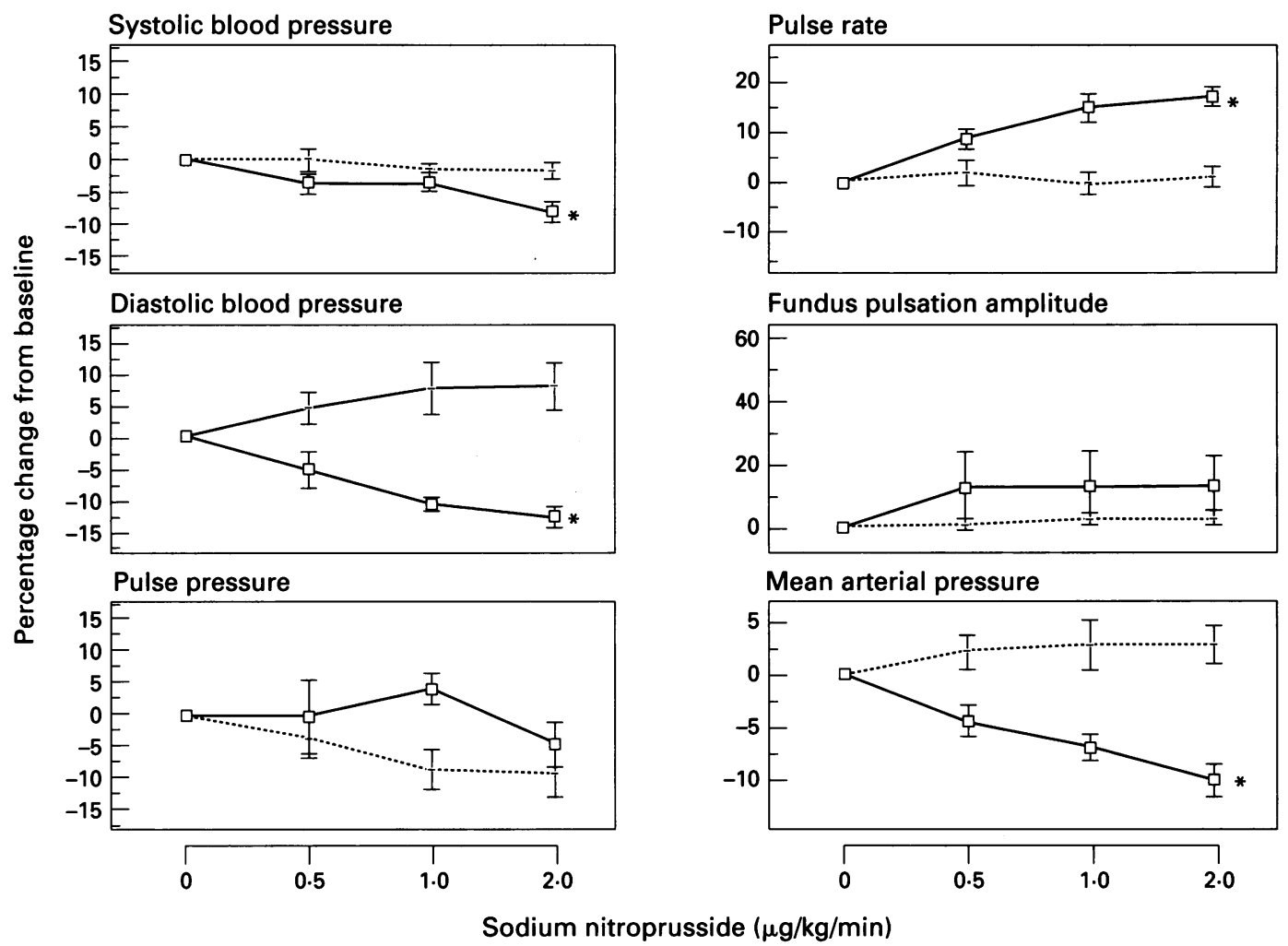

Figure 6 Dose-response relation (solid line) of changes (\%) from baseline measurements of systemic haemodynamics and of fundus pulsations during saline (0) and stepwise infusion of sodium nitroprusside at doses of $0 \cdot 5,1 \cdot 0$, and $2 \cdot 0 \mu \mathrm{g} / \mathrm{kg} / \mathrm{min}$. The broken line presents the results from the placebo study day. The asterisks indicate significant treatment effects versus placebo as calculated by repeated measure ANOVA ( $p<0.05)$. Results are presented as means (SEM).

administration of phenylephrine, the slight reduction of cardiac output and stroke volume as reported by several authors must be taken into account. ${ }^{22} 23$ Hence the lack of effect on fundus pulsation amplitude may be caused by an increase of pulsatile blood flow compared with the total flow or by a local reaction in ocular vessels. The first assumption is supported by the unchanged pulse pressure amplitude in spite of the increased mean arterial pressure during infusion of phenylephrine. Local autoregulative reactions in the choroid are unlikely, ${ }^{9}$ whereas the observation that posterior ciliary arteries spontaneously develop tone $\mathrm{e}^{24}$ indicates an autoregulatory capacity in these prechoroidal arteries. Finally, our results could depend on the change in arterial compliance compared with increased arterial pressure. In the radial artery there is experimental evidence that the higher the arterial pressure, the smaller is the arterial cross section change due to a defined change in blood pressure. ${ }^{25}$ However, the stretch behaviour of a vessel as large as the radial artery will be somewhat different from that of the much smaller choroidal vessels. ${ }^{6}$

Following the administration of sodium nitroprusside no change in stroke volume has been observed in healthy subjects. ${ }^{26}$ However, limited changes in arterial compliance as well as autoregulation in the posterior ciliary arteries again cannot be excluded in our experimental setup. Owing to the increased mean arterial pressure the transmural pressure is high and the smooth muscles relaxed. Hence the elastic behaviour and the tension should rely largely on the passive components of the vessel wall.
Phenylephrine, but not sodium nitroprusside, ${ }^{16}$ slightly decreased IOP. Reduced IOP causes a reduced venous pressure and therefore an increased perfusion pressure in ocular vessels. Yet the transmural pressure changes in choroidal arteries are not relevantly influenced by this mechanism. The small reduction of IOP during infusion of isoproterenol, leading to a decreased venous pressure, is negligible in comparison with the increase in pulse pressure amplitude.

Whether absolute measurements of pulsatile ocular blood flow are feasible with our instrument requires further investigation. It is well known that, as a result of injection of blood flow in the choroid, the ocular volume as well as the IOP changes. ${ }^{27}$ This fact is used for the measurement of ocular blood flow with a pneumatic tonometer. ${ }^{28}$ Hence, it is likely that the cornea, which we use as a reference surface, shows small pulsatile movements as well. Estimation of these movements from the Friedenwald equation ${ }^{29}$ is difficult owing to the different elastic behaviour of the cornea and the sclera. ${ }^{30} \mathrm{We}$ can only assume that the influence of cornea pulsations is small, as we observed big differences in fundus pulsation amplitudes at various retinal measurement points, when the corneal

Table 1 Effect of $0.8 \mu \mathrm{g} / \mathrm{min}$ isoproterenol (Iso) and of 2 $\mu \mathrm{g} / \mathrm{kg} / \mathrm{min}$ phenylephrine (Phe) on fundus pulsation amplitude (FPA in units of $\mathrm{N} 2)$ and IOP ( $\mathrm{mm} \mathrm{Hg}$ ) in eight healthy volunteers. Results are presented as mean (SD).

\begin{tabular}{lrlrr}
\hline & Saline & Iso & \multicolumn{1}{l}{ Saline } & \multicolumn{1}{l}{ Phe } \\
\hline IOP & $13 \cdot 1(2 \cdot 2)$ & $12 \cdot 6(2 \cdot 5)$ & $12 \cdot 6(2 \cdot 7)$ & $12 \cdot 0(2 \cdot 8)$ \\
FPA & $8 \cdot 8(2 \cdot 1)$ & $13.4(3 \cdot 2)$ & $8 \cdot 9(2 \cdot 7)$ & $8 \cdot 8(2 \cdot 3)$ \\
\hline
\end{tabular}


reflection always originates from the same point. ${ }^{15}$

We conclude that the measurements of fundus pulsations in healthy volunteers is a highly reproducible method. The sensitivity of the method to detect changes in ocular blood flow following administration of isoproterenol was excellent even at the lowest dose. However, in situations where the transmural pressure is high and the smooth muscles relaxed or the smooth muscle tension is high, we do not yet know whether changes in choroidal blood flow can be recorded with our method with satisfactory sensitivity. Nevertheless, measurements of fundus pulsations may prove to be a novel diagnostic tool for the assessment of choroidal circulation in vascular diseases of the eye as high transversal resolution is achieved.

The authors thank $\mathrm{Mr} \mathrm{H}$ Sattmann for constructing the electronics of the laser interferometer. Excellent support during the measurements by $\mathrm{H}$ Breiteneder, $\mathrm{RN}, \mathrm{B}$ Brückner, $\mathrm{RN}$, A Molnar, RN, and B Monitzer, RN, is acknowledged. Financial support was given by the Österreichische Nationalbank grant No 4733 .

1 Bill A. Blood circulation and fluid dynamics in the eye. Physiol Rev 1975; 55: 383-417.

2 Ahmed J, Braun RD, Dunn R, Linsenmeier RA. Oxygen distribution in the macaque retina. Invest Ophthalmol Vis Sci 1993; 34: 516-21.

3 Novotny HR, Alvis DL. A method of photographing fluorescence in circulating blood in the human retina. Circulation 1961; 24: 82-6.

4 Richard G. Die klinische Anwendung der Videoangiographie der Retina. Klin Monatsbl Augenheilkd 1984; 185 119-22.

5 Alm A, Bill A. The oxygen supply of the retina: II. Effects of high intraocular pressure and of increased arterial carbon dioxide tension on uveal and retinal blood flow in cats: study with radioactively labelled microspheres including flow determination in brain and some other tissues. Acta Physiol Scand 1972; 84: 306-19.

6 Alm A, Bill A. Ocular and optic nerve blood flow at norma and increased intraocular pressure in monkeys (Macaca irus): a study with radioactively labelled microspheres including flow determinations in brain and some other tissues. Exp Eye Res 1973; 15: 15-29.

7 Friedman E. Choroidal blood flow: pressure flow relationships. Arch Ophthalmol 1970; 83: 95-9.

8 Yu DY, Alder VA, Cringle SJ, Brown MJ. Choroidal blood flow measured in the dog eye in vivo and in vitro by local hydrogen clearance polarography: validation of a technique and effect to raised intraocular pressure. Exp Eye Res 1988; 46: 289-303.
9 Kiel JW, Sheperd AP. Autoregulation of choroidal blood flow in the rabbit. Invest Ophthalmol Vis Sci 1992; 33: 2399-410.

10 Caro CG, Pedley TJ, Schroter RC, Seed WA. The mechanics of circulation. Oxford: Oxford University Press, 1978.

11 Tardy Y, Meister J, Perret F, Brunner HR, Arditi M. Non-invasive estimate of the mechanical properties of peripheral arteries from ultrasonic and photoperipheral arteries from ultrasonic and photoplethysmographic

12 Glucksberg MR, Dunn R. Direct measurement of retinal microvascular pressures in the live, anesthetized cat. Microvasc Res 1993; 45: 158-65.

13 Maepea $O$. Pressures in the anterior ciliary arteries, choroidal veins and choriocapillaris. Exp Eye Res 1992; 54: 731-6.

14 Fine BS. Ocular histology. Hagerstown: Harper and Row, 1979.

15 Schmetterer L, Lexer F, Unfried C, Sattmann H, Fercher A. Topical measurement of fundus pulsations. Opt Eng 1995; 34: 711-6.

16 Elliott WJ, Karnezis TA, Silverman RA, Geanon J, Tripathi RC, Murphy MB. Intraocular pressure increases with fenoldopam, but not nitroprusside, in hypertensive humans. Clin Pharmacol Ther 1991; 49: 285-93.

17 Nathanson JA. Nitrovasodilators as a new class of ocular hypotensive agents. F Pharmacol Exp Ther 1992; 260: 956-65.

18 James CB, Trew DR, Clark K, Smith SE. Factors influencing the ocular pulse-axial length. Graefes Arch Clin Exp Ophthalmol 1991; 229: 341-4.

19 Fryczkowski AW, Sherman MD, Walker J. Observations on the lobular organization of the human choriocapillaris. Int Ophthalmol 1991; 15: 109-20.

20 Trew DR, James CB, Thomas HL, Sutton R, Smith SE. Factors influencing the ocular pulse - the heart rate. Graefes Arch Clin Exp Ophthalmol 1991; 229: 553-6.

21 Grajewski AL, Ferrari-Dileo G, Feuer WJ, Anderson DR. Beta-adrenergic responsiveness of choroidal vasculature. Ophthalmology 1991; 89: 989-95.

22 Shenker Y, Bates E, Egan B, Hammoud J, Grekin R. Effect of vasopressors on arterial natriuretic factor and hemodynamic function in humans. Hypertension 1988; 12: 20-5.

23 Stamler JS, Loh E, Roddy M, Currie KE, Creager MA. Nitric oxide regulates basal systemic and pulmonary vascular resistance in healthy humans. Circulation 1994; 89: cular resistar

24 Nyborg NCB, Nielsen PJ. The level of spontaneous myogenic tone in isolated human posterior ciliary arteries decreases with age. Exp Eye Res 1990; 51: 711-5.

25 Perret F, Mooser V, Hayoz D, Tardy Y, Meister J, Etienne $\mathrm{JD}$, et al. Evaluation of arterial compliance-pressure curves. Hypertension 1991; 18 (Suppl II): 77-83.

26 Merillon JP, Motte G, Aumont MC, Prasquier R, Gourgon $R$. Study of left ventricular pressure-volume relations during nitroprusside infusion in human subjects without during nitroprusside infusion in human subjects withour

27 Silver DM, Farrell RA, Langham ME, O'Brien V, Schilder P. Estimation of pulsatile ocular blood flow from intraocular pressure. Acta Ophthalmol 1989; 67 (Suppl 191): 14-25.

28 Langham ME, Farrell RA, O'Brien V, Silver DM, Schilder P. Blood flow in the human eye. Acta Ophthalmol 1989; 67 (Suppl 191): 9-13.

29 Friedenwald JS. Retinal vascular dynamics. Am $\mathcal{f}$ Ophthalmol 1934; 17: 387-95.

30 Edmund $\mathrm{C}$. Corneal elasticity and ocular rigidity in normal and keratoconic eyes. Acta Ophthalmol 1988; 66: 134-40. 